\title{
Seasonal and diel variation in the fish assemblage of a Neotropical delta in southern Brazil
}

\author{
Adriana Saccol-Pereira ${ }^{1} \&$ Clarice B. Fialho ${ }^{2}$ \\ 1. Laboratório de Ecologia de Águas Continentais, Departamento de Ecologia e Zoologia, Centro de Ciências Biológicas, Universidade \\ Federal de Santa Catarina, Campus Universitário, Trindade, 88010-970 Florianópolis, SC, Brazil. (a.saccolpereira@yahoo.com.br) \\ 2. Laboratório de Ictiologia, Departamento de Zoologia, Universidade Federal do Rio Grande do Sul, Av. Bento Gonçalves, 9500, $91501-$ \\ 970 Porto Alegre, RS, Brazil.
}

\begin{abstract}
The objective of this study was to identify the patterns of seasonal and diel variation and the most important abiotic factors that influence variation in the fish assemblage of the Delta of the Jacuí River in southern Brazil. Seventy-two samples were collected over a one year period. Water temperature was the abiotic factor with the greatest influence on the distribution of the assemblage. The structure of the assemblage exhibited significant changes in terms of species abundance and biomass during the year, with the greatest abundance and biomass being observed during the autumn. There was no significant difference between day and night in terms of abundance, but biomass was significantly greater during the night than during the day.
\end{abstract}

KEYWORDS. Fish ecology, freshwater fish, biological rhythm, circadian rhythm, Patos Lagoon.

RESUMO. Variação sazonal e circadiana na assembleia de peixes em delta Neotropical no sul do Brasil. O objetivo deste estudo foi identificar o padrão de variação sazonal e circadiana e os principais fatores abióticos que influenciam a estrutura da assembleia de peixes do delta do rio Jacuí, região sul do Brasil. Foram realizadas 72 amostragens durante o período de um ano. A distribuição da assembleia foi influenciada principalmente pela temperatura da água. A estrutura da assembleia apresentou diferenças significativas na abundância e biomassa das espécies entre as estações do ano e as maiores abundância e biomassa foram apresentadas no outono. Quando os resultados do dia e da noite foram comparados, a abundância não apresentou diferença significativa, entretanto a biomassa da noite foi significativamente maior que a do dia.

PALAVRAS-CHAVE. Ecologia de peixes, peixes de água doce, ritmo biológico, ritmo circadiano, laguna dos Patos.

The temporal and spatial variations of fish assemblages are influenced by both biotic and abiotic factors. Even in adjacent biotopes, these assemblages exhibit a high degree of variation which is dependent upon the relationships between each species intrinsic characteristics and environmental variables such as resource accessibility and availability (JACKSON et al., 2001; OKADA et al., 2003).

Several authors have emphasized the importance of studying the structure of fish assemblages as a tool for understanding the environment and for establishing relationships between their structures and environmental factors (RICKLEFS, 1987; JUNK et al., 1989; TEJERINA-GARRO et al., 1998; LOREAU et al., 2001; GRANADO-LORENCIO et al., 2005; Piana et al., 2006). These studies have investigated ecological mechanisms that act on assemblages (e.g. competition, reproduction, parasitism and predation) and evaluated interactions with environmental variables (e.g. dissolved oxygen, temperature and seasonality). It is therefore evident that the question of which environmental and ecological factors have a significant influence on the structure of the assemblage in a given location is considered important within the field of ecology (Welcomme, 1979; SCHLOSSER, 1982; TOWNSEND \& HILDREW, 1994; BROWN, 2000; SCHEINER \& WILLIG, 2008).

The structure of a local assemblage may undergo seasonal and diel variations. In a considerable part of the Neotropical region, seasonal variation has been described in studies investigating the differences between flood and dry periods. These studies have demonstrated that there is greater availability of food and increased species abundance during flood periods, leading them to consider this seasonal phenomenon to be the main modulating the structure of the biota (Agostinho et al., 1995; Matthews, 1998; Lake, 2003; Freitas \& Garcez, 2004; MAgalHÃEs et al., 2007; Sousa \& Freitas, 2008).

However, no studies could be located that have focused on the structure of the fish assemblage in Neotropical environments exhibiting both periods of high and low rainfall and strong temperature variations. Temperature is a limiting factor and higher or lower temperatures can favour the presence or absence of certain species, affecting the distribution and composition of the community (MAGNUSON et al., 1979; JaUREGUizar et al., 2003; Wolter, 2007; Cussac et al., 2009).

Furthermore, species-specific diel variations can mean that the abundance of individuals and species in a given habitat changes over the course of 24-hours, as determined by feeding activities (RoOKER \& DeNNIS, 1991; Piet \& GuRuge, 1997), predation and the need to escape from predators (Wright, 1989; COPP \& JURAJDA, 1993; Burrows et al., 1994; GiBSON et al., 1998; Grossman et al., 1998). According to Lowe-McConnell (1999) the presence of predators and large species at night, while small species protect themselves in the vegetation, is a common observation in Neotropical rivers.

This article describes a study in which fishes were sampled systematically in a Delta located in the 
Neotropical region of southern Brazil with the objective to investigate whether seasonal and diel variation is related to abiotic factors $(\mathrm{pH}$; Secchi transparency; dissolved oxygen; air and water temperature; rainfall and water column depth).

\section{MATERIAL AND METHODS}

Study area. The Jacuí River Delta is located in southern Brazil and receives water from the Jacuí, Gravataí, Caí and Sinos rivers, forming a flooded freshwater area of approximately 22 thousand hectares comprising several islands and a network of channels and wetlands that provide the inflow to the Guaíba Lake, which itself flows out to the Patos Lagoon and on to the Atlantic Ocean (Fig. 1). The Delta is located in a region with a subtropical humid climate and has water temperature that varies from $11^{\circ} \mathrm{C}$ to $24^{\circ} \mathrm{C}$ over the course of the year, and a mean air temperature of $19.5^{\circ} \mathrm{C}$, which can vary by up to $9^{\circ} \mathrm{C}$ during daylight hours (MALuF, 2000; FARIA \& LERSCH, 2001). Annual rainfall is less than $1500 \mathrm{~mm}$ and the lowest rainfall is observed between December and February (MALuF, 2000).

Field sampling. Sampling was carried out monthly from October 2004 to September 2005, at three locations that were considered replicates of each other: Saco da Alemoa (SA) (29059'56.5' S, 51 $\left.{ }^{\circ} 14^{\prime} 53.4^{\prime \prime} \mathrm{W}\right)$, Saco do Quilombo (SQ) (29 $\left.58^{\circ} 31^{\prime \prime} \mathrm{S}, 5^{\circ} 15^{\prime} 53.2^{\prime \prime} \mathrm{W}\right)$, and Saco do Ferraz (SF) (300'34.8'S, 51 14 '41.3"W) (Fig. 1). All of these sampling stations are bays with a depth of more than $1.2 \mathrm{~m}$, bushy vegetation, grass, and macrophytes (Eichhornia spp.) growing along the banks (OLIVEIRA \& PORTO, 1999).

The following physical and chemical measurements were taken monthly at each sampling area: $\mathrm{pH}$, Secchi transparency, dissolved oxygen and air and water temperature. Additionally, monthly data on rainfall and water column depth were obtained from the eighth district of the Instituto Nacional de Meteorologia and from the
Superintendência de Portos e Hidrovias, respectively. We used several gillnets for each sampling area, with mesh sizes of $1.5,2.5,3.5,4.5$ and $6.0 \mathrm{~cm}$ between adjacent knots and with dimensions of $20 \times 1.5 \mathrm{~m}$, making a total area of $150 \mathrm{~m}^{2}$. The nets were arranged in sequence, perpendicular to the shore for a period of 24 hours. Nets were set at $6 \mathrm{pm}$ and were checked at 6am on the following day. All individuals collected during this period were considered nocturnal, while individuals collected between $6 \mathrm{am}$ and $6 \mathrm{pm}$ were considered diurnal. Environmental data was used to define the seasons as follows, spring: September, October and November; summer: December, January and February, autumn: March, April and May and winter: June, July and August.

The individuals collected were classified according to taxonomic keys and were measured (mm), and weighed $(\mathrm{g})$. Voucher species were deposited in the ichthyologic collection of the Laboratório de Ictiologia, Departamento de Zoologia, Instituto de Biociências, Universidade Federal do Rio Grande do Sul, Brazil, under the following accession numbers: UFRGS 0088, 0188, 4190, 5059, 5172, 5838, 6366, 6707, 6727, 6989, 7114, 7576-7585, 7588-7590, 7592-7594, 7595, $7656,8190,8259,8643,8916,8917$.

Data analysis. In order to describe the main species distribution tendencies and to relate them to environmental factors, monthly means were calculated from the absolute numbers of individuals captured at the three sampling areas and, independent of diel variation, correlated with the abiotic data using canonical correspondence analysis (CCA), performed with CANOCO version 4.5 software (TER BRAAK, 1995).

The data were natural log-transformed $(\mathrm{Ay}+\mathrm{B})$ and the "downweighting of rare species" option was selected in order to ensure that where few individuals of a given species had been collected, this would not affect the final result. The significance of environmental factors was estimated using the "vif" function (variance inflation factor), which detects co-linearity between variables,

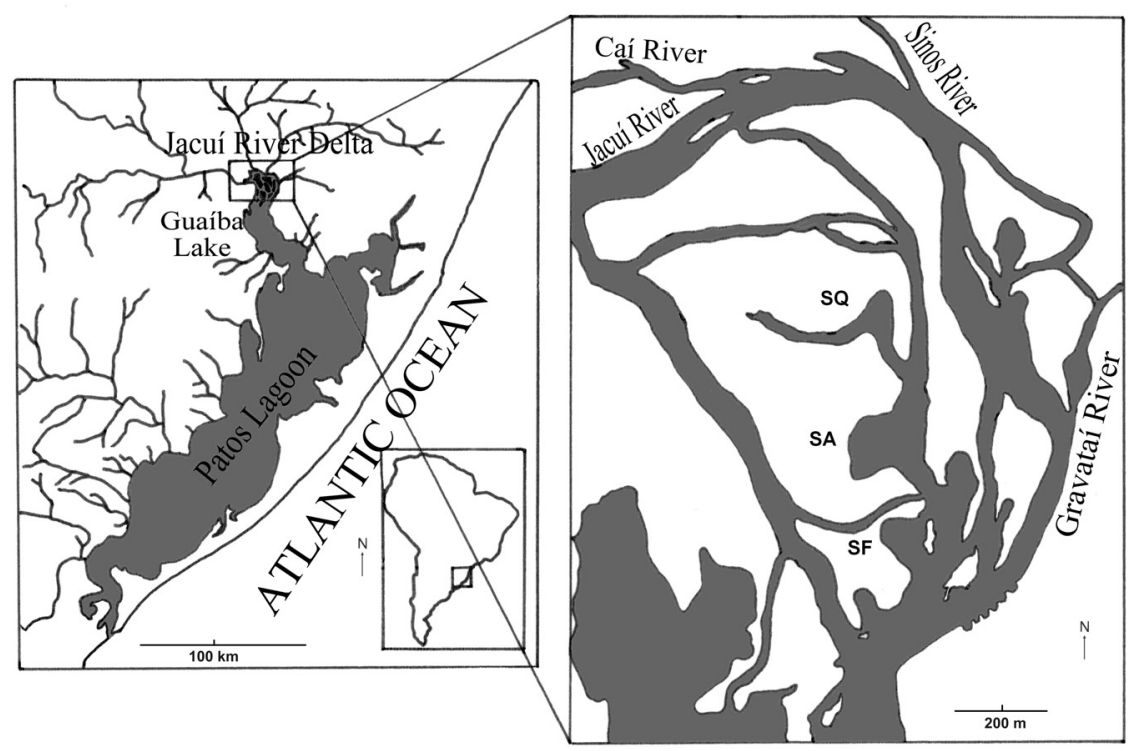

Figure 1. Location of the Jacuí River Delta, southern Brazil, with sample areas: Saco do Quilombo (SQ), Saco da Alemoa (SA) and Saco do Ferraz (SF). 
where variables whose vif $>20$ were defined as redundant and removed from the analysis (MYERs, 1990; HAIR et al., 1998). Statistical significance was estimated using the Monte Carlo permutation test $\left(10^{4}\right.$ unrestricted permutations) with the significance level set at $P \leq 0.05$. The null hypothesis was that species abundance did not correlate with environmental variables.

Principal coordinates analysis (PCA, TER BRAAK, 1995) was performed using a matrix of abundance data for each species, for each season, and for the day and night periods, in order to represent the pattern of associations between species composition and seasons and day/night. Data were natural log-transformed (Ay + B) and the downweighting rare species option was once more selected in order to avoid the influence of rare species leading to biased results. Detrended correspondence analyses (DCA, TER BRAAK, 1995) was performed in order to verify the length of the gradient (< $3 \mathrm{SD}$ ) and, on the basis of the DCA results, a linear PCA model was selected, in line with standard practice (TER BRAAK, 1995). Ordination analyses were carried out using the computer program CANOCO version 4.5 .

Hypotheses were tested using analysis of variance with randomization (MANLY, 1991) in order to verify whether there were significant diel differences between species abundance at seasonal stations. A multivariate analysis based on randomization and bootstrap nonparametric re-sampling methods was performed (MANLY, 1991; Crowley, 1992; PodAni, 1994; Pillar, 1998; 1999). Data were transformed $[\log (\mathrm{x}+1)]$, and the Euclidian distance was used as measure of similarity. The randomization test was performed through 1000 interactions and the main criterion adopted was the sum of the square of the distances between groups. The significance level used for all analyses was $P \leq 0.05$ and the null hypothesis tested was that the composition of these species does not change in relation to seasonal variations and is independent of day or night. The MULTIV computer program, version 2.4.2 (PILLAR, 2006), was used to transform the data and perform the similarity measurements and randomization tests.

The constancy $(c)$ for each species was calculated using the number of months and the period (day/night) during which the species was collected. Each species were classified as frequent $(c>50 \%)$, accessory $(25 \% \leq c$ $\leq 50 \%)$, or accidental $(c<25 \%)$ (DAJOZ, 1983).

Indices of richness (MARGALEF, 1951), diversity $H^{\prime}$ (ln), and evenness J' (PIELOU, 1966, 1975) were calculated using the Divers computer program (PEREZ-LoPEZ \& SOLAFernandez, 1993). MULTIV version 2.4.2 (PiLlar, 2006) was used to compare results with analysis of variance (one-way ANOVA), by season and sampling period (day/ night).

\section{RESULTS}

A total of 3862 individuals were collected, distributed across 34 species (Tab. I). Cyphocharax voga (Hensel, 1870), Astyanax fasciatus (Cuvier, 1819),
Parapimelodus nigribarbis (Boulenger, 1889), Pachyurus bonariensis Steindachner, 1879, Corydoras paleatus (Jenyns, 1842), Loricariichthys anus (Valenciennes, 1835), Pimelodus maculatus Lacepède, 1803 and Hoplosternum littorale (Hancock, 1828) accounted for $80 \%$ of the total catch in terms of number of individuals, while C. voga, L. anus, P. bonariensis, P. maculatus, Hoplias malabaricus (Bloch, 1794) and $H$. littorale accounted for $80 \%$ of the total biomass (Tab. II).

With relation to the significance of the environmental factors, the variables "air temperature" and "depth of the water column" were considered redundant (vif > 20) and were removed from the analysis. The environmental variable measurements are given in table III and the results of the canonical correspondence analysis (Tab. IV; Fig. 2) based on these measurements demonstrated that water temperature was the environmental variable with the greatest influence on the distribution of species. The results of this analysis were significant and the environmental factors measured explained $59.2 \%$ of the variation in abundance. The first and second ordination axes were predominantly correlated with the variables "water temperature" and "rainfall" (Tab. V).

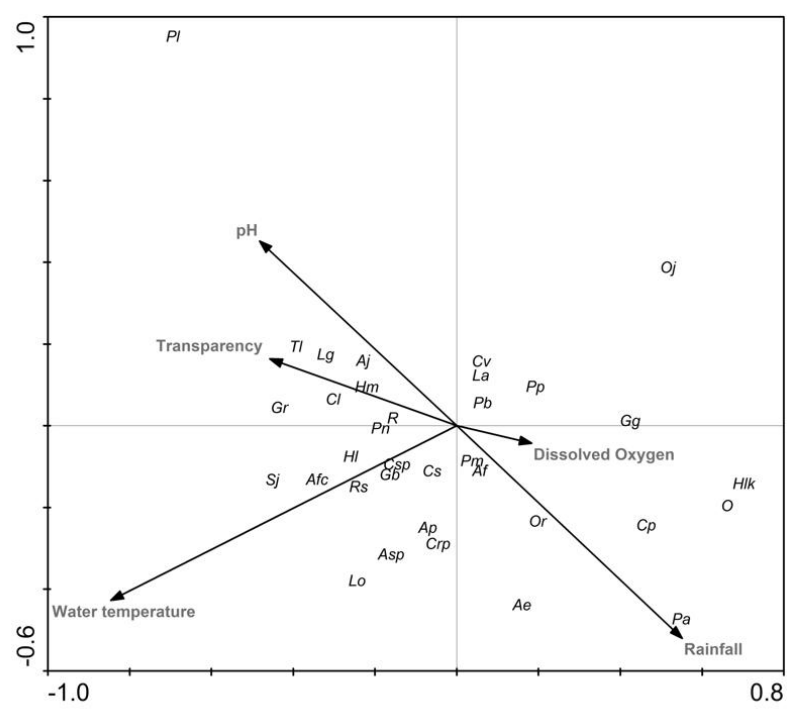

Figure 2. Pattern in fish assemblages distribution among environmental variables based on canonical correspondence analysis (CCA) in the Jacuí River Delta, RS, Brazil, from October 2004 to September 2005 (Ap, Acestrorhyncus pantaneiro; Ae, Astyanax eigenmanniorum; Af, Astyanax fasciatus; Aj, Astyanax jacuhiensis; Asp, Astyanax sp.; Afc, Australoheros facetus; Cs, Charax stenopterus; $\mathrm{Cp}$, Corydoras paleatus; $\mathrm{Cl}$, Crenicichla lepidota; Crp, Crenicichla punctata; $\mathrm{Cv}$, Cyphocharax voga; Csp, Cyphocharax spilotus; Gb, Geophagus brasiliensis; Gg, Gymnogeophagus gymnogenys; Gr, Gymnogeophagus rhabdotus; Hm, Hoplias malabaricus; H1, Hoplosternum littorale; H1k, Hyphessobrycon luetkenii; Lo, Leporinus obtusidens; La, Loricariichthys anus; Lg, Lycengraulis grossidens; O, Odontesthes sp.; Oj, Oligosarcus jenynsii; Or, Oligosarcus robustus; $\mathrm{Pb}$, Pachyurus bonariensis; $\mathrm{Pn}$, Parapimelodus nigribarbis; Pa, Pimelodella australis; Pm, Pimelodus maculatus; Pp, Platanichthys platana; P1, Prochilodus lineatus; R, Rhamdia; Rs, Rineloricaria strigilata; $\mathrm{Sj}$, Schizodon jacuiensis; T1, Trachelyopterus lucenai). 
Table I. Individuals (n) collected in the Jacuí River Delta, southern Brazil, from October 2004 to September 2005 in every season and day period and their respective ecological indices.

\begin{tabular}{|c|c|c|c|c|c|c|c|c|c|c|c|}
\hline \multirow[t]{2}{*}{ Taxa } & \multirow[t]{2}{*}{ Abbrev. } & \multicolumn{2}{|c|}{ Spring } & \multicolumn{2}{|c|}{ Summer } & \multicolumn{2}{|c|}{ Autumn } & \multicolumn{2}{|c|}{ Winter } & \multicolumn{2}{|c|}{ Constancy (\%) } \\
\hline & & Day & Night & Day & Night & Day & Night & Day & Night & Day & Night \\
\hline \multicolumn{12}{|l|}{ ATHERINIFORMES } \\
\hline \multicolumn{12}{|l|}{ Atherinopsidae } \\
\hline Odontesthes sp. & $O$ & 3 & & & & & & 1 & & & 16.7 \\
\hline \multicolumn{12}{|l|}{ CHARACIFORMES } \\
\hline \multicolumn{12}{|l|}{ Acestrorhynchidae } \\
\hline Acestrorhynchus pantaneiro Menezes, 1992 & $A p$ & 2 & & 2 & & 2 & 2 & & & 50 & 16.7 \\
\hline \multicolumn{12}{|l|}{ Anostomidae } \\
\hline Leporinus obtusidens Valenciennes, 1837 & Lo & & & & 1 & 4 & 1 & & & 16.7 & 16.7 \\
\hline Schizodon jacuiensis Bergmann, 1988 & $S j$ & 1 & 2 & 3 & 2 & 4 & 1 & & & 33.3 & 25 \\
\hline Characidae & & & & & & & & & & & \\
\hline Astyanax eigenmanniorum (Cope, 1894) & $A e$ & 1 & 1 & 1 & & 5 & 1 & & & 41.7 & 8.3 \\
\hline Astyanax fasciatus (Cuvier, 1819) & Af & 43 & 56 & 66 & 41 & 198 & 36 & 20 & 12 & 100 & 100 \\
\hline Astyanax jacuhiensis (Cope, 1894) & $A j$ & 7 & & 10 & 7 & 2 & & 1 & & 50 & 25 \\
\hline Astyanax sp. & Asp & 1 & & 2 & & 7 & & & & 33.3 & \\
\hline Charax stenopterus (Cope, 1894) & Cs & & & 2 & 4 & 1 & 3 & 1 & 1 & 25 & 25 \\
\hline Hyphessobrycon luetkenii (Boulenger, 1887) & $H l k$ & 3 & 5 & & & & & & 4 & 8.3 & 16.7 \\
\hline Oligosarcus jenynsii (Günther, 1864) & $O j$ & 1 & 1 & & & 21 & 3 & 15 & 43 & 33.3 & 50 \\
\hline Oligosarcus robustus Menezes, 1969 & $\mathrm{Or}$ & 3 & 3 & 2 & 3 & 12 & 21 & & 6 & 33.3 & 50 \\
\hline Curimatidae & & & & & & & & & & & \\
\hline Cyphocharax spilotus (Vari, 1987) & Csp & 22 & 4 & 16 & 4 & 8 & & 3 & 1 & 91.6 & 50 \\
\hline Cyphocharax voga (Hensel, 1870) & $C v$ & 103 & 79 & 139 & 20 & 244 & 264 & 110 & 203 & 100 & 91.6 \\
\hline Erythrinidae & & & & & & & & & & & \\
\hline Hoplias malabaricus (Bloch, 1794) & $\mathrm{Hm}$ & 12 & 11 & 10 & 26 & 3 & 13 & 1 & 6 & 66.7 & 91.6 \\
\hline Prochilodontidae & & & & & & & & & & & \\
\hline Prochilodus lineatus (Valenciennes, 1837) & $P l$ & 1 & & & & & & & & 8.3 & \\
\hline CLUPEIFORMES & & & & & & & & & & & \\
\hline Engraulidae & & & & & & & & & & & \\
\hline Lycengraulis grossidens (Agassiz, 1829) & $L g$ & 6 & 2 & 2 & 4 & 3 & & 2 & & 66.7 & 33.3 \\
\hline Clupeidae & & & & & & & & & & & \\
\hline Platanichthys platana (Regan, 1917) & $P p$ & 2 & 1 & & & & & 1 & & 16.7 & 16.7 \\
\hline PERCIFORMES & & & & & & & & & & & \\
\hline Cichlidae & & & & & & & & & & & \\
\hline Australoheros facetus (Jenyns, 1842) & $A f_{c}$ & & & 1 & & 1 & & & & 16.7 & \\
\hline Geophagus brasiliensis (Quoy \& Gaimard, 1824) & $G b$ & 4 & 1 & 16 & 2 & 19 & 4 & 2 & 4 & 66.7 & 58.3 \\
\hline Gymnogeophagus gymnogenys Hensel, 1870 & $G g$ & 4 & 3 & & & 8 & 3 & 7 & 3 & 58.3 & 33.3 \\
\hline Gymnogeophagus rhabdotus Hensel, 1870 & $G r$ & 3 & 1 & 4 & 4 & 8 & 1 & & & 41.7 & 25 \\
\hline Crenicichla lepidota Heckel, 1840 & $\mathrm{Cl}$ & 4 & 1 & 12 & & & & & & 41.7 & 8.3 \\
\hline Crenicichla punctata Hensel, 1870 & Crp & 5 & & & & 3 & 2 & & & 33.3 & 16.7 \\
\hline Sciaenidae & & & & & & & & & & & \\
\hline Pachyurus bonariensis Steindachner, 1879 & $P b$ & 15 & 9 & 25 & 11 & 117 & 42 & 51 & 45 & 91.6 & 100 \\
\hline SILURIFORMES & & & & & & & & & & & \\
\hline Auchenipteridae & & & & & & & & & & & \\
\hline $\begin{array}{l}\text { Trachelyopterus lucenai } \\
\text { Bertoletti, Pezzi da Silva \& Pereira, } 1995\end{array}$ & $T l$ & 3 & 10 & & 2 & & 3 & & & 8.3 & 41.7 \\
\hline Callichthyidae & & & & & & & & & & & \\
\hline Corydoras paleatus (Jenyns, 1842) & $C p$ & 2 & 9 & & & 95 & 66 & 15 & 109 & 41.7 & 50 \\
\hline Hoplosternum littorale (Hancock, 1828) & $\mathrm{Hl}$ & 10 & 12 & 11 & 58 & 14 & 39 & & 4 & 66.7 & 91.6 \\
\hline Loricariidae & & & & & & & & & & & \\
\hline Loricariichthys anus (Valenciennes, 1835) & $L a$ & 14 & 28 & 47 & 44 & 29 & 24 & 23 & 57 & 100 & 91.6 \\
\hline Rineloricaria strigilata (Hensel, 1868) & Rs & & & & 2 & & & & 1 & & 8.3 \\
\hline Heptapteridae & & & & & & & & & & & \\
\hline Rhamdia spp. & $R$ & & 1 & 7 & 13 & 2 & 2 & 3 & 4 & 33.3 & 66.7 \\
\hline Pimelodella australis Eigenmann, 1917 & $P a$ & & & & & 1 & & & & 8.3 & \\
\hline Pimelodidae & & & & & & & & & & & \\
\hline Parapimelodus nigribarbis (Boulenger, 1889) & $P n$ & 22 & 57 & 66 & 14 & 150 & 41 & 18 & 7 & 91.6 & 83.3 \\
\hline Pimelodus maculatus LaCepède, 1803 & $P m$ & 31 & 36 & 44 & 13 & 58 & 28 & 10 & 25 & 91.6 & 91.6 \\
\hline Total & & 328 & 333 & 488 & 275 & 1019 & 600 & 284 & 535 & & \\
\hline No. of species & & 28 & 23 & 22 & 20 & 27 & 22 & 18 & 18 & & \\
\hline Diversity & & 2.45 & 2.27 & 2.29 & 2.4 & 2.25 & 2.01 & 1.92 & 1.92 & & \\
\hline Richness & & 4.66 & 3.78 & 3.39 & 3.38 & 3.75 & 3.28 & 3 & 2.7 & & \\
\hline Eveness & & 0.73 & 0.72 & 0.74 & 0.8 & 0.68 & 0.65 & 0.68 & 0.66 & & \\
\hline No. of samples & & 9 & 9 & 9 & 9 & 9 & 9 & 9 & 9 & & \\
\hline
\end{tabular}


Table II. Biomass (g) of individuals collected in the Jacuí River Delta, southern Brazil, from October 2004 to September 2005 in each season (day and night).

\begin{tabular}{|c|c|c|c|c|c|c|c|c|c|}
\hline \multirow[t]{2}{*}{ Taxa } & \multirow[t]{2}{*}{ Abbrev. } & \multicolumn{2}{|c|}{ Spring } & \multicolumn{2}{|c|}{ Summer } & \multicolumn{2}{|c|}{ Autumn } & \multicolumn{2}{|c|}{ Winter } \\
\hline & & Day & Night & Day & Night & Day & Night & Day & Night \\
\hline \multicolumn{10}{|l|}{ ATHERINIFORMES } \\
\hline \multicolumn{10}{|l|}{ Atherinopsidae } \\
\hline Odontesthes sp. & $O$ & 173.9 & & & & & & 20.42 & \\
\hline \multicolumn{10}{|l|}{ CHARACIFORMES } \\
\hline \multicolumn{10}{|l|}{ Acestrorhynchidae } \\
\hline Acestrorhyncus pantaneiro Menezes, 1992 & $A p$ & 151.3 & & 154.79 & & 38.0 & 242.0 & & \\
\hline \multicolumn{10}{|l|}{ Anostomidae } \\
\hline Leporinus obtusidens Valenciennes, 1837 & Lo & & & 91.6 & & 642.0 & 268.0 & & \\
\hline Schizodon jacuiensis Bergmann, 1988 & $S j$ & 19.2 & 149.7 & 230.2 & 205.0 & 514.0 & 155.0 & & \\
\hline \multicolumn{10}{|l|}{ Characidae } \\
\hline Astyanax eigenmanniorum (Cope, 1894) & Ae & 6.6 & 9.3 & 10.0 & & 60.0 & 8.0 & & \\
\hline Astyanax fasciatus (Cuvier, 1819) & Af & 519.8 & 598.8 & 790.6 & 518.9 & 2278.0 & 441.0 & 223.01 & 133.21 \\
\hline Astyanax jacuhiensis (Cope, 1894) & $A j$ & 86.8 & & 375.0 & 68.3 & 54.0 & & 6.8 & \\
\hline Astyanax sp. & Asp & 9.8 & & 24.2 & & 85.0 & & & \\
\hline Charax stenopterus (Cope, 1894) & Cs & & & 13.0 & 29.0 & 6.0 & 19.0 & 7.0 & 8.0 \\
\hline Hyphessobrycon luetkenii (Boulenger, 1887) & $H l k$ & 22.3 & 30.1 & & & & & & 27.63 \\
\hline Oligosarcus jenynsii (Günther, 1864) & $O j$ & 42.0 & 55.0 & & & 1643.0 & 231.0 & 1075.0 & 3085.0 \\
\hline Oligosarcus robustus Menezes, 1969 & Or & 169.6 & 293.0 & 39.0 & 272.0 & 1645.0 & 1701.0 & & 684.0 \\
\hline \multicolumn{10}{|l|}{ Curimatidae } \\
\hline Cyphocharax spilotus (Vari, 1987) & Csp & 266.1 & 60.1 & 213.2 & 68.6 & 158.0 & & 45.35 & 10.8 \\
\hline Cyphocharax voga (Hensel, 1870) & $C v$ & 8359.9 & 8269.9 & 16695.9 & 2192.3 & 30308.5 & 35001.8 & 13076.0 & 27845.98 \\
\hline \multicolumn{10}{|l|}{ Erythrinidae } \\
\hline Hoplias malabaricus (Bloch, 1794) & $\mathrm{Hm}$ & 2372.2 & 3286.00 & 1911.2 & 8490.3 & 688.0 & 3958.0 & 321.0 & 2076.0 \\
\hline Prochilodontidae & & & & & & & & & \\
\hline Prochilodus lineatus (Valenciennes, 1837) & $P l$ & 250.0 & & & & & & & \\
\hline CLUPEIFORMES & & & & & & & & & \\
\hline Engraulidae & & & & & & & & & \\
\hline Lycengraulis grossidens (Agassiz, 1829) & $L g$ & 304.4 & 78.4 & 57.6 & 89.1 & 165.0 & & 75.0 & \\
\hline Clupeidae & & & & & & & & & \\
\hline Platanichthys platana (Regan, 1917) & $P p$ & 10.2 & 10.3 & & & & & 7.47 & \\
\hline PERCIFORMES & & & & & & & & & \\
\hline Cichlidae & & & & & & & & & \\
\hline Australoheros facetus (Jenyns, 1842) & $A f c$ & & & 84.9 & & 80.0 & & & \\
\hline Geophagus brasiliensis (Quoy \& Gaimard, 1824) & 4) $G b$ & 258.6 & 98.0 & 1073.0 & 244.0 & 1012.0 & 245.0 & 136.0 & 342.0 \\
\hline Gymnogeophagus gymnogenys Hensel, 1870 & $G g$ & 95.2 & 58.0 & & & 193.0 & 94.0 & 235.0 & 97.0 \\
\hline Gymnogeophagus rhabdotus Hensel, 1870 & $G r$ & 156.0 & 41.9 & 111.2 & 104.4 & 545.0 & 41.0 & & \\
\hline Crenicichla lepidota Heckel, 1840 & $\mathrm{Cl}$ & 410.1 & 26.4 & 382.3 & & & & & \\
\hline Crenicichla punctata Hensel, 1870 & $C r p$ & 442.1 & & & & 255.0 & 116.0 & & \\
\hline Sciaenidae & & & & & & & & & \\
\hline Pachyurus bonariensis Steindachner, 1879 & $\mathrm{~Pb}$ & 1223.9 & 652.2 & 1803.1 & 894.0 & 9809.0 & 3947.0 & 6024.0 & 5209.5 \\
\hline SILURIFORMES & & & & & & & & & \\
\hline Auchenipteridae & & & & & & & & & \\
\hline Trachelyopterus lucenai & $T l$ & 326.0 & 676.9 & & 164.6 & & 257.0 & & \\
\hline Bertoletti, Pezzi da Silva \& Pereira 1995 & & & & & & & & & \\
\hline Callichthyidae & & & & & & & & & \\
\hline Corydoras paleatus (Jenyns, 1842) & $C p$ & 20.0 & 78.0 & & & 829.0 & 578.0 & 129.0 & 825.0 \\
\hline Hoplosternum littorale (Hancock, 1828) & $H l$ & 709.3 & 1570.5 & 1548.4 & 6691.1 & 1398.0 & 4589.0 & & 563.0 \\
\hline Loricariidae & & & & & & & & & \\
\hline Loricariichthys anus (Valenciennes, 1835) & $L a$ & 1763.0 & 3710.5 & 5028.37 & 5390.5 & 3633.0 & 3492.0 & 2959.0 & 7944.0 \\
\hline Rineloricaria strigilata (Hensel, 1868) & Rs & & & & 18.0 & & & & 20.0 \\
\hline Heptapteridae & & & & & & & & & \\
\hline Rhamdia spp. & $R$ & & 456.0 & 2089.4 & 3238.5 & 563.0 & 760.0 & 1600.0 & 1286.0 \\
\hline Pimelodella australis Eigenmann, 1917 & $P a$ & & & & & 11.0 & & & \\
\hline Pimelodidae & & & & & & & & & \\
\hline Parapimelodus nigribarbis (Boulenger, 1889) & $P n$ & 376.7 & 1004.5 & 1076.5 & 290.4 & 3168.0 & 825.0 & 357.0 & 95.0 \\
\hline Pimelodus maculatus LaCepède, 1803 & $P m$ & 3984.0 & 3620.9 & 4006.7 & 1318.6 & 6799.0 & 2663.0 & 920.0 & 2918.0 \\
\hline Total & & 22529.1 & 24834.4 & 37718.5 & 30379.2 & 66579.5 & 59631.8 & 27217.0 & 53170.1 \\
\hline No. of species & & 28 & 23 & 22 & 20 & 27 & 22 & 18 & 18 \\
\hline No. of samples & & 9 & 9 & 9 & 9 & 9 & 9 & 9 & 9 \\
\hline
\end{tabular}


In contrast with the majority of species observed, the overall tendency of the species Oligosarcus jenynsii (Günther, 1864), C. paleatus and L. anus was to exhibit increased abundance during the winter, particularly during the night. Conversely, Acestrorhynchus pantaneiro Menezes, 1992, Schizodon jacuiensis Bergmann, 1988, Astyanax eigenmanniorum (Cope, 1894), Gymnogeophagus rhabdotus Hensel, 1870 and Trachelyopterus lucenai Bertoletti, Pezzi da Silva \& Pereira, 1995 were all caught during the other three seasons, but not during winter, leading to lower species richness in the winter.

The number of individuals observed increased during the autumn; in particular, there were greater numbers of $A$. fasciatus, $P$. bonariensis and $P$. nigribarbis during the daytime, while high frequencies of predator species such as Oligosarcus robustus Menezes, 1969, H. malabaricus, T. lucenai, H. littorale and Rhamdia spp. were observed during the nocturnal period (Tab. I).

Abundance and biomass were significantly higher during the winter (Tab. II) than during the summer and the lowest abundance and biomass were observed during the spring.

Analysis of variance with randomization demonstrated that, independent of diel variation, autumn was the season with the greatest abundance of species, significantly different from winter $(P=0.03)$, spring $(P=$ $0.04)$ and summer $(P=0.005)$. Winter abundance was significantly greater than summer abundance $(P=0.01)$. No significant differences were detected in species abundance when the differences between numbers of individuals observed during the day and during the night were analyzed independent of season.
With relation to the differences in biomass between seasons, independent of diel variation, autumn exhibited the greatest biomass, with a statistically significant difference in relation to winter $(P=0.007)$, summer $(P=$ $0.006)$, and spring $(P=0.05)$. Winter biomass was significantly greater than summer biomass $(P=0.003)$ and biomass was significantly lower during spring than during the summer $(P=0.01)$. When diurnal biomass was compared with nocturnal biomass, independent of season, the sum of the nocturnal individuals' mass was significantly greater $(P=0.01)$ than that of the diurnal individuals (Fig. 3).

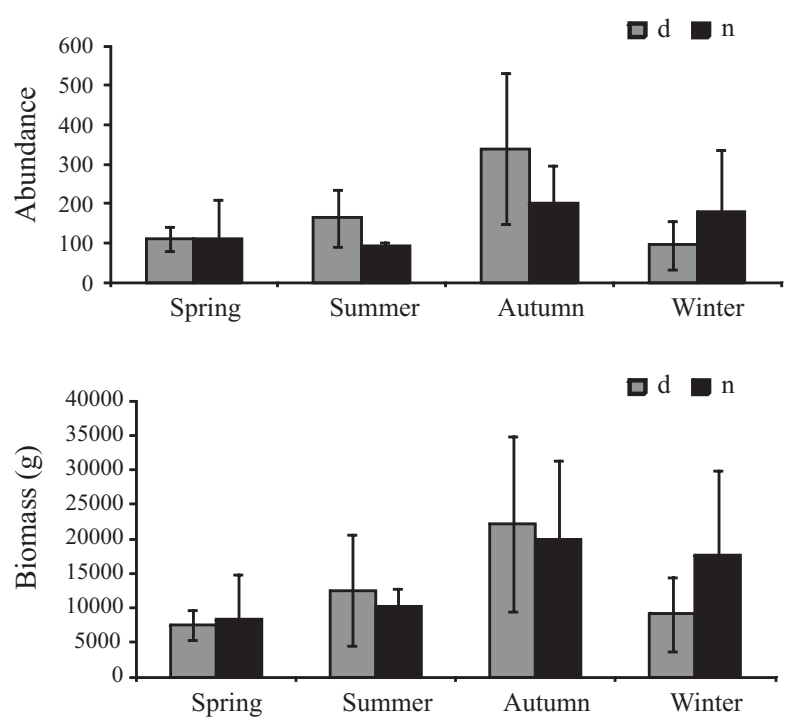

Figure 3. Seasonal abundance (n) and biomass (g) (mean \pm SD) and period of collection (day/night) in the Jacuí River Delta, southern Brazil, from October 2004 to September 2005.

Table III. Mean $( \pm$ SD) of environmental variables in the sample areas (SA; SQ; SF) in the Jacuí River Delta, southern Brazil, from October/2004 to September/2005.

\begin{tabular}{|c|c|c|c|c|c|c|c|c|c|c|c|c|}
\hline & Oct & Nov & Dec & Jan & $\mathrm{Feb}$ & Mar & Apr & May & Jun & Jul & Aug & Sep \\
\hline $\begin{array}{l}\text { Water } \\
\text { temperature }\end{array}$ & $21.0 \pm 0.1$ & $24.1 \pm 0.3$ & $19.3 \pm 0.6$ & $24.7 \pm 2.3$ & $21.7 \pm 0.6$ & $23.3 \pm 2.3$ & $21.0 \pm 0.1$ & $18.5 \pm 0.5$ & $17.4 \pm 0.4$ & $13.3 \pm 0.4$ & $14.0 \pm 0.1$ & $18.0 \pm 1.0$ \\
\hline $\mathrm{pH}$ & $7.16 \pm 0.1$ & $7.2 \pm 0.1$ & $6.6 \pm 0.4$ & $6.7 \pm 0.5$ & $6.7 \pm 0.6$ & $6.9 \pm 0.7$ & $6.7 \pm 0.1$ & $6.5 \pm 0.2$ & $6.9 \pm 0.1$ & $6.9 \pm 0.1$ & $6.8 \pm 0.1$ & $6.4 \pm 0.1$ \\
\hline $\begin{array}{l}\text { Secchi } \\
\text { transparency }(\mathrm{cm})\end{array}$ & $53.6 \pm 4.9$ & $33.6 \pm 16.8$ & $25.0 \pm 4.5$ & $20.3 \pm 7.6$ & $7.7 \pm 2.5$ & $24.7 \pm 6.6$ & $38 \pm 3.6$ & $15.3 \pm 1.5$ & $14 \pm 2.6$ & $20 \pm 0.1$ & $20 \pm 0.1$ & $0.3 \pm 9.4$ \\
\hline $\begin{array}{l}\text { Dissolved oxygen } \\
(\mathrm{mg} / \mathrm{L})\end{array}$ & $3.7 \pm 0.4$ & $6.4 \pm 2.0$ & $9.0 \pm 1.7$ & $8.0 \pm 0.1$ & $8.6 \pm 0.1$ & $9.0 \pm 1.7$ & $8.0 \pm 0.1$ & $7.3 \pm 1.1$ & $9.3 \pm 2.8$ & $7.1 \pm 1.6$ & $11.0 \pm 0.1$ & $5.0 \pm 1.7$ \\
\hline $\begin{array}{l}\text { Rainfall } \\
(\mathrm{mm})\end{array}$ & 76.2 & 117.6 & 33.7 & 28.8 & 41.0 & 141.3 & 145.8 & 153.7 & 15.2 & 57.7 & 155.9 & 164.3 \\
\hline $\begin{array}{l}\text { Rainfall (mm) } \\
\text { 1961-1992 }\end{array}$ & 114.3 & 104.2 & 101.2 & 100.1 & 108.6 & 104.4 & 86.1 & 94.6 & 132.7 & 121.7 & 140.0 & 139.5 \\
\hline
\end{tabular}

Table IV. Summary of the results of canonical correspondence analysis (CCA) based on five environmental variables measures in the Jacuí River Delta, southern Brazil, from October 2004 to September 2005 (* Global test).

\begin{tabular}{lcc}
\hline & Axis 1 & Axis 2 \\
\hline Eigenvalues $(\lambda)$ & 0.164 & 0.062 \\
Pearson's correlations (species-environment) & 0.964 & 0.972 \\
Cumulative percentage variance of species data $(\%)$ & 28.5 & 39.2 \\
Cumulative percentage variance of species-environment relation (\%) & 48.1 & 2.387 \\
Monte Carlo test & $F$ & 0.01 \\
& $P$ & $0.002 *$
\end{tabular}


The first two axes of the ordination analysis (PCA) explained $65.9 \%$ of seasonal species variability. Axis 1 $(42.3 \%)$ demonstrated that, with the exception of diurnal winter data and nocturnal autumn data there was separation between the species collected during the day and during the night, with greater richness during the daytime (Fig. 4).

When species were arranged in order of biomass, the two first axes explained $52.7 \%$ of the observed variation, with $30.1 \%$ explained by axis 1 . Although species richness was greater during the diurnal period when compared with the nocturnal period, the largest species, with the greatest biomass, were observed during the night, meaning that total biomass was greater at night (Fig. 5).

Table I lists the results of classifying the species by constancy of occurrence $(c)$ and also gives the results for richness, diversity $H^{\prime}(\ln )$ and evenness $J^{\prime}$. Analysis of variance of the indices of richness, diversity $H^{\prime}$ and evenness $J$ ' did not detect significant seasonal variation (Tab. VI) or significant diel variation (diversity $P=0.74$, evenness $P=1$, richness $P=0.38$ ).

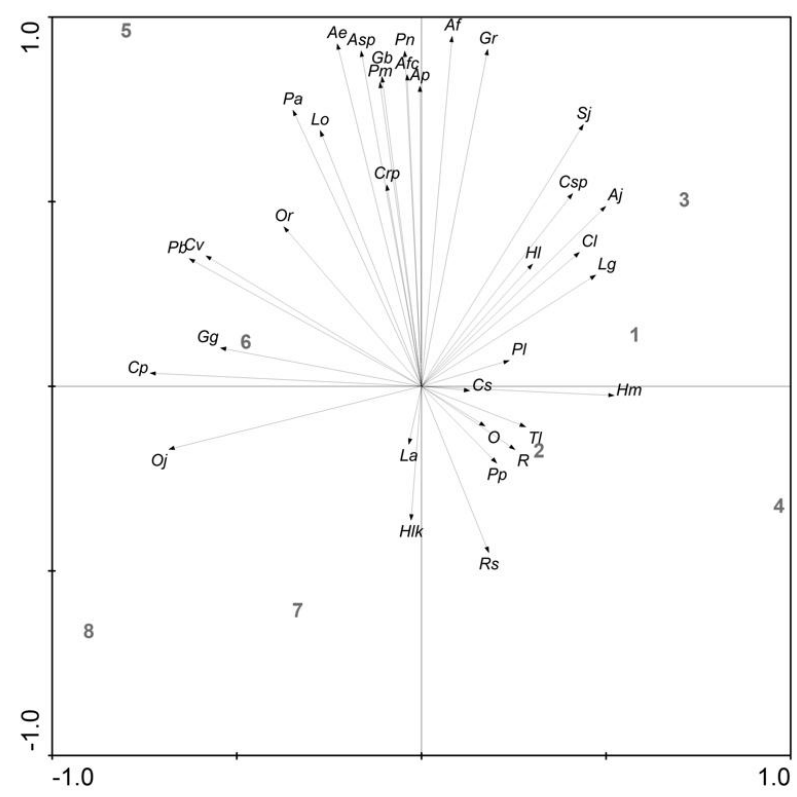

Figure 4. Ordination of the species (number of individuals) in relation to season (day/night) resulting from principal components analysis (PCA) applied to abundance (n) data matrix in the Jacuí River Delta, RS, Brazil, from October 2004 to September 2005 (1, spring day; 2, spring night; 3 , summer day; 4, summer night; 5 , autumn day; 6 , autumn night; 7 , winter day; 8 , winter night; Ap, Acestrorhyncus pantaneiro; Ae, Astyanax eigenmanniorum; Af, Astyanax fasciatus; Aj, Astyanax jacuhiensis; Asp, Astyanax sp.; Afc, Australoheros facetus; Cs, Charax stenopterus; Cp, Corydoras paleatus; $\mathrm{Cl}$, Crenicichla lepidota; Crp, Crenicichla punctata; $\mathrm{Cv}$, Cyphocharax voga; Csp, Cyphocharax spilotus; Gb, Geophagus brasiliensis; Gg, Gymnogeophagus gymnogenys; Gr, Gymnogeophagus rhabdotus; Hm, Hoplias malabaricus; $\mathrm{H1}$, Hoplosternum littorale; Hlk, Hyphessobrycon luetkenii; Lo, Leporinus obtusidens; La, Loricariichthys anus; Lg, Lycengraulis grossidens; $\mathrm{O}$, Odontesthes sp.; $\mathrm{Oj}$, Oligosarcus jenynsii; Or, Oligosarcus robustus; $\mathrm{Pb}$, Pachyurus bonariensis; $\mathrm{Pn}$, Parapimelodus nigribarbis; $\mathrm{Pa}$, Pimelodella australis; $\mathrm{Pm}$, Pimelodus maculatus; $\mathrm{Pp}$, Platanichthys platana; $\mathrm{Pl}$, Prochilodus lineatus; R, Rhamdia spp.; Rs, Rineloricaria strigilata; $\mathrm{Sj}$, Schizodon jacuiensis; Tl, Trachelyopterus lucenai).
Table V. Matrix of correlation among environmental variables measures and abundance of individuals collected in the Jacuí River Delta, southern Brazil, from October 2004 to September 2005.

\begin{tabular}{lcr}
\hline & \multicolumn{2}{c}{ Principal Components } \\
\hline Variables & Axis 1 & Axis 2 \\
Water temperature $\left({ }^{\circ} \mathrm{C}\right)$ & -0.8150 & -0.4154 \\
$\mathrm{pH}$ & -0.4648 & 0.4391 \\
Dissolved Oxygen $(\mathrm{mg} / \mathrm{L})$ & 0.1769 & -0.0417 \\
Secchi Transparency $(\mathrm{cm})$ & -0.4408 & 0.1588 \\
Rainfall $(\mathrm{mm})$ & 0.5312 & -0.5051 \\
\hline
\end{tabular}

Table VI. Ecological indexes between seasons in the Jacuí River Delta, southern Brazil, from October 2004 to September 2005.

\begin{tabular}{lccc}
\hline Contrasts & Diversity & Evenness & Richness \\
\hline spring x summer & $\mathrm{p}$ & $\mathrm{p}$ & $\mathrm{p}$ \\
spring x autumn & 0.41 & 0.32 & 0.34 \\
spring x winter & 0.32 & 0.32 & 0.34 \\
summer x autumn & 0.3 & 0.34 & 0.33 \\
summer x winter & 0.31 & 0.34 & 0.33 \\
autumn x winter & 0.34 & 1 & 0.32 \\
\hline
\end{tabular}

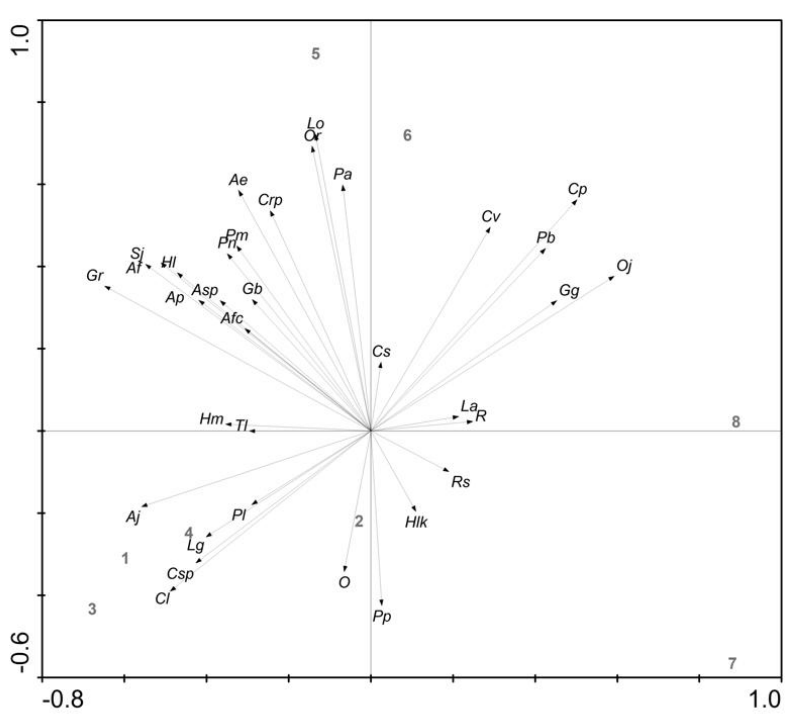

Figure 5. Ordination of the species (biomass) in relation to season (day/night) resulting from principal components analysis (PCA) applied to total biomass (g) data matrix in the Jacuí River Delta, RS, Brazil, from October 2004 to September 2005 (1, spring day; 2, spring night; 3 , summer day; 4 , summer night; 5 , autumn day; 6, autumn night; 7, winter day; 8; winter night; Ap, Acestrorhyncus pantaneiro; Ae, Astyanax eigenmanniorum; Af, Astyanax fasciatus; Aj, Astyanax jacuhiensis; Asp, Astyanax sp.; Afc, Australoheros facetus; Cs, Charax stenopterus; Cp, Corydoras paleatus; $\mathrm{Cl}$, Crenicichla lepidota; $\mathrm{Crp}$, Crenicichla punctata; $\mathrm{Cv}$, Cyphocharax voga; Csp, Cyphocharax spilotus; Gb, Geophagus brasiliensis; Gg, Gymnogeophagus gymnogenys; Gr, Gymnogeophagus rhabdotus; Hm, Hoplias malabaricus; H1, Hoplosternum littorale; Hlk, Hyphessobrycon luetkenii; Lo, Leporinus obtusidens; La, Loricariichthys anus; Lg, Lycengraulis grossidens; O, Odontesthes sp.; $\mathrm{Oj}$, Oligosarcus jenynsii; Or, Oligosarcus robustus; $\mathrm{Pb}$, Pachyurus bonariensis; $\mathrm{Pn}$, Parapimelodus nigribarbis; $\mathrm{Pa}$, Pimelodella australis; $\mathrm{Pm}$, Pimelodus maculatus; Pp, Platanichthys platana; Pl, Prochilodus lineatus; R, Rhamdia spp.; Rs, Rineloricaria strigilata; $\mathrm{Sj}$, Schizodon jacuiensis; Tl, Trachelyopterus lucenai). 


\section{DISCUSSION}

According to Lowe-McConnell (1999), it is very common for Ostariophysi to dominate in Neotropical rivers, in particular Characiformes and Siluriformes, and this is what was observed in the Jacuí River Delta. Cyphocharax voga exhibited the greatest biomass and abundance during all periods, followed by P. nigribarbis, C. paleatus, $L$. anus, and P. maculatus. Overall, the fish that make up the assemblage in the Jacuí River Delta also occur frequently in the Patos Lagoon system (Malabarba, 1989).

One factor that could contribute to the biomass and abundance of the species mentioned above is the fact that the Delta has a muddy bottom with large quantities of debris resulting from the confluence of rivers from different areas (FARIA \& LERSCH, 2001). This feature would encourage a local increase in the abundance and biomass of detritivorous species such as C. voga, $P$. nigribarbis, C. paleatus, L. anus, and P. maculatus (HARTZ \& BARBIERI, 1993; Yossa \& ARAúJo-Lima, 1998; Delariva \& Agostinho, 2001; Fugi et al., 2001; Lima-Junior \& Goitein, 2004; GRANADO-LoRENCIO et al., 2005; CARDONE et al., 2006). According to Grenouillet et al. (2002), species abundance is intimately related to the availability of food sources.

However, in common with the description of Patos Lagoon published by PEREIRA (1994), seasonal factors have a significant influence on the distribution of individuals in the Delta. During periods of heavy rain and higher water levels, an elevated abundance of freshwater fish was observed in Patos Lagoon, which led researchers to raise the hypothesis that some species may be being carried towards the lagoon (GARCIA \& VIEIRA, 2001; GARCIA et al., 2003). Notwithstanding, abundance of species and biomass in the Delta also increased in line with rainfall. MACHADO-AlLISON (1990) made the observation that increases in the abundance of species are to be expected during periods with increased rainfall because of the greater availability of food sources.

In contrast with species in flooded areas, where the structure of the assemblage is mainly influenced by the flood pulse (WinEMILLER et al., 2000; SúAREZ et al., 2001), variations in the ichthyofauna in the Delta were mainly influenced by water temperature. This pattern is similar to that observed in other areas with subtropical climates (JAUREGUIZAR et al., 2003; AdAMS et al., 2004).

Previous studies carried out with three different species of the genus Astyanax (BERTACO et al., 1998) and with P. nigribarbis (BERTACO \& BECKER, 2000) in Guaíba Lake have reported low abundance during periods with low temperatures, and related this decrease to individuals seeking protected areas in deeper waters. Individuals engaging in such migration would avoid collection by gillnets, which may also have occurred in the study described here.

Alternatively, the largest number of individuals caught in autumn, resulting in greater abundance and biomass, could be a result of increasing movement and feeding activity of individuals in the period before the breeding season of most species. Furthermore, it has been demonstrated that many fish feed with greater intensity during the periods before and after their reproductive season (BARBIERI et al., 1982; SCHLOSSER, 1982; HARTZ et al., 1996; TRIPE \& GUY, 1999).

The majority of species inhabiting the coastal regions of southern Brazil begin their reproductive cycles during the spring (FialHo et al., 1996; Nunes et al., 2004; Marques et al., 2007; Milani \& Fontoura, 2007) and the fact that the greatest richness and lowest abundance and biomass was observed during this period could be related to a high number of juveniles in species assemblages. These juveniles would not be collected by gillnets due to their small size.

In addition to seasonal influences, several fish exhibit diel variation in their habitat use, varying in biomass, richness and abundance when day and night are compared (LOWE-McConNeLl, 1964; WINEMILLER, 1989; Gaudreau \& Bosclair, 1998; Yu \& Peters, 2003; Wolter \& FReYhoF, 2004; OKun et al., 2005). Although the number of individuals did not significantly differ between day and night, biomass was significantly higher during the night, which is probably linked to frequent collection of large predator species such as $H$. littorale, $H$. malabaricus, O. robustus, Rhamdia spp. and $T$. lucenai at night. This result is in contrast with the abundance data, where a large number of small individuals were observed during the day.

Finally, in common with tropical regions, where fish assemblages are influenced mainly by rainfall, in this subtropical area rainfall also plays a role. However, this study supports the view that temperature-related seasonal and diel variation is the most important factor impacting the distribution of the fish assemblage in the Jacuí River Delta.

Acknowledgments. The authors are grateful to the Parque Estadual Delta do Jacuí staff for their assistance, especially to Clemente da Rosa for his help in the boat. We would also like to thank the staff at the Laboratorio de Ictiologia (UFRGS), and in particular L. R. Malabarba, R. Hirano, J. Ferrer, A. P. Dufech, T. S. Dias, J. Giora, P. Milani, F. Schneck and R. Pereira for their valuable help in the field, with the statistical analysis and for reviewing this manuscript. This study was supported by the Conselho Nacional de Desenvolvimento Científico e Tecnológico (140879/2004-8).

\section{REFERENCES}

Adams, S.; Warren JR, M. L. \& HaAG, W. R. 2004. Spatial and temporal patterns in fish assemblages of upper coastal plain streams, Mississippi, USA. Hydrobiologia 528:45-61.

Agostinho, A. A.; Vazzoler, A. E. A. M. \& Thomaz, S. M. 1995. The High river Paraná basin: limnological and ichthyological aspects. In: Tundisi, J. G.; Bicudo, C. E. M. \& MatsumuraTundisI, T. eds. Limnology in Brazil. Rio de Janeiro, ABC/ SBL. p.59-103.

Barbieri, G.; Verani, J. R. \& Barbieri, M. C. 1982. Dinâmica quantitativa da nutrição de Hoplias malabaricus (Bloch, 1974), na represa do Lobo (Brotas-Itirapina/SP) (Pisces, Erythrinidae). Revista Brasileira de Biologia 42(2):295-302.

Bertaco, V. A. \& Becker, F. G. 2000. Observações sobre a abundância de Parapimelodus nigribarbis (Boulenger, 1889) (Pimelodidae) no lago Guaíba, Rio Grande do Sul, Brasil. Comunicações do Museu de Ciências e Tecnologia da PUCRS, Série Zoologia, 13(2):185-195.

Bertaco, V. A.; Lucena Z. M. S. \& Becker, F. G. 1998. Variação espacial e temporal na abundância de Astyanax bimaculatus e Astyanax fasciatus (Characidae) no lago Guaíba, Rio Grande do Sul, Brasil. Comunicações do Museu de Ciências e Tecnologia da PUCRS, Série Zoologia, 11:61-89. 
Brown, L. R. 2000. Fish communities and their associations with environmental variables, lower San Joaquin River Drainage, California. Environmental Biology of Fishes 57:251-269.

Burrows, M. T.; Gibson, R. N.; Robb, L. \& Comely, C. A. 1994. Temporal patterns of movement in juvenile flatfishes and their predators: underwater television observations. Journal of Experimental Marine Biology and Ecology 177:251268 .

Cardone, I. B.; Lima-Junior, S. E. \& Goitein, R. 2006. Diet and capture of Hypostomus strigaticeps (Siluriformes, Loricariidae) in a small Brazilian stream: relationship with limnological aspects. Brazilian Journal of Biology 66(1A):25-33.

Copp, G. H. \& Jurajda, P. 1993. Do small riverine fish move inshore at night? Journal of Fish Biology 43(Suppl. A):229241 .

Crowley, P. H. 1992. Resampling methods for computation intensive data analysis in ecology and evolution. Annual Review of Ecological Systems 23:405-447.

Cussac, V. E.; Fernández, D. A.; Gomez, S. E. \& López, H. L. 2009. Fishes of southern South America: a story driven by temperature. Fish Physiology and Biochemistry 35:2942.

Dajoz, R. 1983. Ecologia Geral. Petrópolis, Vozes. 472p.

Delariva, R. L. \& Agostinho, A. A. 2001. Relationship between morphology and diets of six neotropical loricariids. Journal of Fish Biology 58:832-847.

Faria, C. M. \& Lersch, E. C. 2001. Monitoramento das águas do Delta e foz dos rios formadores do Guaíba. Revista Ecos Pesquisa 2(5):7-42.

Fialho, C. B.; Verani, J. R.; Peret, A. C. \& Bruschi Jr., W. 1996. Dinâmica da reprodução de Odontesthes aff. perugiae (Evermann \& Kendall, 1906) da lagoa Emboaba, RS, Brasil (Pisces, Atherinidae). Boletim do Instituto de Pesca 23: $105-115$.

Freitas, C. E. C. \& Garcez, R. C. S. 2004. Fish communities of natural canals between floodplain lakes and SolimõesAmazonas River. Acta Limnologica Brasiliensia 16(3):273-280

Fugi, R.; Agostinho, A. A. \& Hahn, N. S. 2001. Trophic morphology of five benthic-feeding fish species of a tropical floodplain. Revista Brasileira de Biologia 61(1):27-33.

Garcia, A. M. \& Vieira, J. P. 2001. O aumento da diversidade de peixes no estuário da lagoa dos Patos durante o episódio $\mathrm{El}$ Niño 1997-1998. Atlântica 23:85-96.

Garcia, A. M.; Raseira, M. B.; Vieira, J. P.; Winemiller, K. O. \& Grimm, A. M. 2003. Spatiotemporal variation in shallowwater freshwater fish distribution and abundance in a large subtropical coastal lagoon. Environmental Biology of Fishes 68:215-228.

Gaudreau, N. \& Bosclair, D. 1998. The influence of spatial heterogeneity on the study of fish horizontal daily migration. Fisheries Research 35:65-73.

Gibson, R. N.; Pihl, L.; Burrows, M. T.; Modin, J.; Wennhage, H. \& Nickell, L. A. 1998. Diel movements of juvenile plaice Pleuronectes platessa in relation to predators, competitors, food availability and abiotic factors on a microtidal nursery ground. Marine Ecology Progress Series 165:145-159.

Granado-Lorencio, C.; Araújo-Lima, C. R. M. \& Lobón-Cerviá, J. 2005. Abundance - distribution relationships in fish assembly of the Amazonas floodplain lakes. Ecography 28(4):515-520.

Grenoulllet, G.; Pont, D. \& Seip, K. 2002. Abundance and species richness as a function of food resources and vegetation structure: juvenile fish assemblages in rivers. Ecography 25:641-650.

Grossman, G. D.; Ratajczak Jr, R. E.; Crawford, M. \& Freeman, M. C. 1998. Assemblage organization in stream fishes: effects of environmental variation and interspecific interactions. Ecological Monographs 68:395-420.

Hair, J. F. J.; Anderson, R. E.; TAtham, R. L. \& Black, W. C. 1998. Multivariate data analyses. New Jersey, Prentice Hall. $552 \mathrm{p}$.

Hartz, S. M. \& BARbieri, G. 1993. Dinâmica quantitativa da alimentação de Cyphocarax voga (Hensel, 1869) da lagoa Emboaba, RS, Brasil (Characiformes, Curimatidae). Comunicações do Museu de Ciências e Tecnologia da PUCRS, Série Zoologia, 6:63-74.
Hartz, S. M.; Martins, A. \& Barbieri, G. 1996. Dinâmica da alimentação e dieta de Oligosarcus jenynsii (Gunther, 1864) na lagoa Caconde, Rio Grande do Sul, Brasil (Teleostei, Characidae). Boletim do Instituto de Pesca 23:21-29.

Jackson, D. A.; Peres-Neto, P. R. \& Olden, J. D. 2001. What controls who is where in freshwater fish communities - the roles of biotic, abiotic and spatial factors. Canadian Journal of Fisheries and Aquatic Sciences 58:157-170.

Jaureguizar, A. J.; Menni, R.; Guerrero, R. \& Lasta, C. 2003. Environmental factors structuring fish communities of the Rio de la Plata estuary. Fisheries Research 66(2-3):195211

Junk, W. J.; Bayley, P. B. \& Sparks, R. E. 1989. The flood pulse concept in river-floodplain systems. Canadian Journal of Fisheries and Aquatic Sciences 106:110-127.

LAKE, P. S. 2003. Ecological effects of perturbation by drought in flowing water. Freshwater Biology 48:1161-1172.

Lima-Junior, S. E. \& Goitein, R. 2004. Diet and feeding activity of Pimelodus maculatus (Osteichthyes, Pimelodidae) in the Piracicaba river (state of São Paulo, Brazil) - the effect of seasonality. Boletim do Instituto de Pesca 30(2):135-140.

Loreau, M.; Naeem, S.; Inchausti, P.; Bengtsson, J.; Grime, J. P.; Hector, A.; Hooper, D. U.; Huston, M. A.; Raffaelli, D.; Schmid, B.; Tilman, D. \& Wardle, D. A. 2001. Biodiversity and ecosystem functioning: current knowledge and future challenges. Science 294:804-808.

Lowe-McConnell, R. H. 1964. The fishes of the Rupununi savanna district of British Guiana, South America. Journal of the Linnean Society 45:103-144.

1999. Estudos ecológicos de comunidades de peixes tropicais. São Paulo, EDUSP. 534p.

Machado-Allison, A. 1990. Ecologia dos peixes das áreas inundáveis dos Llanos da Venezuela. Interciencia 15:411-423.

Magalhães, M. F.; Beja, P.; Schlosser I. J. \& Collares-Pereira, M. J. 2007. Effects of multi-year droughts on fish assemblages of seasonally drying Mediterranean streams. Freshwater Biology 52(8):1494-1510.

Magnuson, J. J.; Crowder, L. B. \& Medvick, P. A. 1979. Temperature as an Ecological Resource. American Zoologist 19(1):331-343.

Malabarba, L. R. 1989. Histórico sistemático e lista comentada das espécies de peixes de água doce do sistema da laguna dos Patos, Rio Grande do Sul, Brasil. Comunicações do Museu de Ciência e Tecnologia da PUCRS, Série Zoologia, 2(8): 107-179.

Maluf, J. R. T. 2000. Nova classificação climática do estado do Rio Grande do Sul. Revista Brasileira de Agrometeorologia 8(1): 141-150.

Manly, B. F. J. 1991. Randomization and Monte Carlo Methods in biology. London, Chapman \& Hall. 455p.

Margalef, R. 1951. Diversidad de especies en comunidades naturales. Publicaciones del Instituto de Biologia Aplicada e Barcelona 9:5-27.

Marques, C. S.; Braun, A. S. \& Fontoura, N. F. 2007. Estimativa de tamanho de primeira maturação a partir de dados de IGS: Oligosarcus jenynsii, Oligosarcus robustus, Hoplias malabaricus, Cyphocharax voga, Astyanax fasciatus (Characiformes), Parapimelodus nigribarbis, Pimelodus maculatus, Trachelyopterus lucenai, Hoplosternum littorale, Loricariichthys anus (Siluriformes) e Pachyurus bonariensis (Perciformes) no lago Guaíba e laguna dos Patos, RS. Biociências 15(2):230-256.

Matthews, W. J. 1998. Patterns in freshwater ecology. New York, Chapman \& Hall. 756p.

Milani, P. C. \& Fontoura, N. F. 2007. Diagnóstico da pesca artesanal na lagoa do Casamento, sistema nordeste da laguna dos Patos: uma proposta de manejo. Biociências 15(1):82125 .

Myers, R. H. 1990. Classical and modern regression with applications. Belmont, Duxbury. 488p.

Nunes, D. M.; Pellanda, M. \& Hartz, S. M. 2004. Dinâmica reprodutiva de Oligosarcus jenynsii e O. robustus (Characiformes, Characidae) na Lagoa Fortaleza, Rio Grande do Sul, Brasil. Iheringia, Série Zoologia, 94(1):5-11.

Okada, E. K. A.; Agostinho, A. A.; Petrere Jr, M. \& Penczak, T. 2003. Factors affecting fish diversity and abundance in drying 
ponds and lagoons in the upper Paraná River basin, Brazil. Ecohydrology \& Hidrobiology 3(1):97-110.

Okun, N.; Mendonça, R. \& Mehner, T. 2005. Diel shifts in community composition and feeding of juvenile fishes in the pelagic area of a large shallow lake. Limnologica 35:70-77.

Oliveira, M. L. A. A. \& Porto, M. L. 1999. Ecologia de Paisagem do Parque Estadual Delta do Jacuí, Rio Grande do Sul, Brasil: Mapa da Cobertura do Solo e da Vegetação, a partir de imagem do LADSAT.TM5. Iheringia, Série Botânica, 52:145-160.

Pereira, L. E. 1994. Variação diurna e sazonal dos peixes demersais na Barra do Estuário da Lagoa dos Patos, RS. Atlântica 16:521.

Pérez-López, F. J. \& Sola-Fernandez, F. M. 1993. DIVERS: Programa para el cálculo de los índices de diversidad. Available at: 〈http://perso.wanadoo.es/jp-1/descargas.htm〉. Accessed on: 20.08.2007.

Piana, P. A.; Gomes, L. C. \& Cortez, E. M. 2006. Factors influencing Serrapinnus notomelas (Characiformes: Characidae) populations in upper Paraná river floodplain lagoons. Neotropical Ichthyology 4(1):81-86.

Pielou, E. C. 1966. The measurement of diversity in different types of biological collections. Journal of Theoretical Biology 13:131-144.

1975. Ecological diversity. New York, John Wiley \& Sons. $165 \mathrm{p}$

Piet, G. J. \& Guruge, A. H. P. W. 1997. Diel variation in feeding and vertical distribution of ten co-occurring fish species: consequences for resource partitioning. Environmental Biology of Fishes 50:293-307.

Pillar, V. D. 1998. Sampling sufficiency in ecological surveys. Abstracta Botanica 22:37-48.

1999. The bootstrapped ordination re-examined. Journal of Vegetation Science 10:895-902.

PillaR, V. P. 2006. MULTIV, Multivariate Exploratory Analysis, Randomization Testing and Bootstrap Resampling Software. Available at: <http://ecoqua.ecologia.ufrgs.br/ecoqua/ software.html>. Accessed on: 10.11.2007.

PodAnI, J. 1994. Multivariate data analysis in ecology and systematics: a methodological guide to the SYN-TAX 5.0 package. The Hague, SPB Academic. $316 \mathrm{p}$.

Ricklefs, R. E. 1987. Community Diversity: relative roles of local and regional processes. Science 235(4785):167-171.

Rooker, J. R. \& Dennis, G. D. 1991. Diel, lunar and seasonal changes in a mangrove fish assemblage off southwestern Puerto Rico. Bulletin of Marine Science 49:684-698.

Scheiner, S. M. \& Willig, M. R. 2008. A general theory of ecology. Theoretical Ecology 1:21-28.

Schlosser, I. J. 1982. Fish Community Structure and Function along two habitat gradients in a headwater stream. Ecological Monographs 52(4):395-414.

Sousa, R. G. C. \& Freitas, C. E. DE C. 2008. The influence of flood pulse on fish communities of floodplain canals in the Middle Solimões River, Brazil. Neotropical Ichthyology 6(2):249255

Súarez, Y. R.; Petrere Jr., M. \& Catella, A. C. 2001. Factors determining the structure of fish communities in Pantanal lagoons (MS, Brazil). Fisheries Management and Ecology 8:173-186.

Tejerina-Garro, F. L.; Fortín, R. \& Rodríguez, M. A. 1998. Fish community structure in relation to environmental variation in floodplain lakes of the Araguaia River, Amazon Basin Environmental Biology of Fishes 51:399-410.

TER BraAk, C. J. F. 1995. Ordination. In: Jongman, R. H. J.; TER Braak, C. J. F. \& van Tongeren, O. F. R. eds. Data Analysis in Community and Landscape Ecology. Cambridge, Cambridge University. p.91-173.

Townsend, C. R. \& Hildrew, A. G. 1994. Species traits in relation to a habitat templet for river systems. Freshwater Biology 31:265-275.

TRIPE, J. A. \& GuY, C. S. 1999. Spatial and temporal variation in habitat and fish community characteristics in a Kansas Flint Hills stream. Ecology of Freshwater Fish 8:216-226.

Welcomme, R. L. 1979. The fisheries ecology of floodplain rivers. London, Longman. $317 \mathrm{p}$

WinEMILlER, K. O. 1989. Ontogenetic diet shifts and resource partitioning among piscivorous fishes in the Venezuelan llanos. Environmental Biology of Fishes 26:177-199.

Winemiller, K. O.; Tarim, S.; Shormann, D. \& Cotner, J. B. 2000. Fish assemblage structure in relation to environmental variation among Brazos River oxbow lakes. Transactions of the American Fisheries Society 129:451-468.

Wolter, C. 2007. Temperature influence on the fish assemblage structure in a large lowland river, the lower Oder River, Germany. Ecology of Freshwater Fish 16(4):493-503.

Wolter, C. \& FreyhoF, J. 2004. Diel distribution patterns of fishes in a temperate large lowland river. Journal of Fish Biology 64:632-642.

Wright, J. M. 1989. Diel variation and seasonal consistency in the fish assemblage of the non-estuarine Sulaibikhat Bay, Kuwait. Marine Biology 102(1):1432-1793.

Yossa, M. I. \& Araújo-Lima, C. A. R. M. 1998. Detritivory in two Amazonian fish species. Journal of Fish Biology 52:11411153.

Yu, S. L. \& Peters, E. 2003. Diel and seasonal abundance of fishes in the Platte River, Nebraska, USA. Fisheries Science 69: $154-160$

Recebido em junho de 2009. Aceito em abril de 2010. ISSN 0073-4721

Artigo disponível em: www.scielo.br/isz 